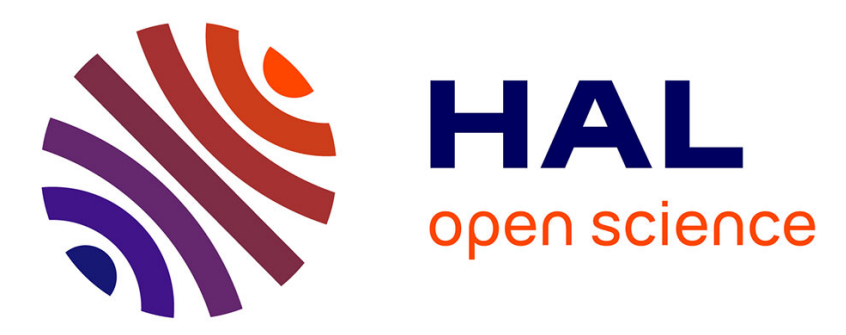

\title{
Étude expérimentale de la relaxation du rubidium en présence d'hélium
}

M. Aymar, M.A. Bouchiat, J. Brossel

\section{To cite this version:}

M. Aymar, M.A. Bouchiat, J. Brossel. Étude expérimentale de la relaxation du rubidium en présence d'hélium. Journal de Physique, 1969, 30 (8-9), pp.619-629. 10.1051/jphys:01969003008-9061900 . jpa-00206826

\section{HAL Id: jpa-00206826 https://hal.science/jpa-00206826}

Submitted on 1 Jan 1969

HAL is a multi-disciplinary open access archive for the deposit and dissemination of scientific research documents, whether they are published or not. The documents may come from teaching and research institutions in France or abroad, or from public or private research centers.
L'archive ouverte pluridisciplinaire HAL, est destinée au dépôt et à la diffusion de documents scientifiques de niveau recherche, publiés ou non, émanant des établissements d'enseignement et de recherche français ou étrangers, des laboratoires publics ou privés. 


\title{
ÉTUDE EXPÉRIMENTALE DE LA RELAXATION DU RUBIDIUM EN PRÉSENGE D’HÉLIUM
}

\author{
Par M. AYMAR, M. A. BOUCHIAT et J. BROSSEL, \\ Faculté des Sciences de Paris, Laboratoire de Spectroscopie Hertzienne de l'E.N.S., associé au C.N.R.S.
}

(Reçu le 24 mars 1969.)

Résumé. - On décrit une étude expérimentale de la relaxation, en présence d’hélium, d'atomes de $\mathrm{Rb}$ polarisés par pompage optique. Des isotopes enrichis de ${ }^{87} \mathrm{Rb},{ }^{85} \mathrm{Rb},{ }^{4} \mathrm{He}$ et ${ }^{3} \mathrm{He}$ ont été utilisés. Différentes causes possibles d'erreurs systématiques sont discutées. On observe que la relaxation de $\left\langle S_{z}\right\rangle$ est caractérisée par deux constantes de temps $\tau$ et $\tau^{\prime}$ dont on a mesuré la variation en fonction de la pression $p$ d'hélium (entre 16 et 700 torrs). A basse pression, $\tau$ et $\tau^{\prime}$ sont associés aux deux premiers modes de diffusion des atomes vers la paroi totalement désorientante (paroi non enduite). On en déduit le coefficient de diffusion de $\mathrm{R} b$ dans l'hélium à la pression atmosphérique et à $300 \mathrm{o}: D_{0}\left(\mathrm{Rb}-{ }^{4} \mathrm{He}\right)=0,42 \pm 0,06 \mathrm{~cm}^{2} / \mathrm{s}$, indépendant de l'isotope $\mathrm{Rb}$ et $D_{0}\left(\mathrm{Rb}-{ }^{3} \mathrm{He}\right) / D_{0}\left(\mathrm{Rb}-{ }^{4} \mathrm{He}\right)=1,25 \pm 0,1$ et on interprète ces résultats.

Aux fortes pressions, au contraire, $\tau$ et $\tau^{\prime}$ sont approximativement les constantes de temps de la relaxation de $\left\langle S_{z}\right\rangle$ induite sous l'effet des chocs contre le gaz (une correction est apportée pour tenir compte de la relaxation sur la paroi après diffusion dans le gaz). On en déduit les sections efficaces $\sigma_{n}\left({ }^{87} \mathrm{Rb}-\mathrm{He}\right)=0,47 \times 10^{-24} \mathrm{~cm}^{2}$ et $\sigma_{n}\left({ }^{85} \mathrm{Rb}-\mathrm{He}\right)=0,27 \times 10^{-24} \mathrm{~cm}^{2}$ pour l'observable $\left\langle I_{z}\right\rangle$, et $\sigma_{e}(\mathrm{Rb}-\mathrm{He})=8,2 \times 10^{-24} \mathrm{~cm}^{2}$ pour l'observable $\left\langle Q_{e}\right\rangle$. L'identité des sections efficaces mesurées pour ${ }^{4} \mathrm{He}$ et ${ }^{3} \mathrm{He}$ est compatible avec 1'hypothèse selon laquelle l'interaction désorientatrice prédominante est d'origine spin-orbite. L'effet du spin nucléaire de ${ }^{3} \mathrm{He}$ est indécelable et donc plus faible qu'il n'était attendu théoriquement. La variation de $\sigma_{n}$, quand on passe de ${ }^{85} \mathrm{Rb}$ à ${ }^{87} \mathrm{Rb}$, est comprise, ainsi que l'identité de $\sigma_{e}$ pour ces deux isotopes. Par contre, pour expliquer le rapport $\sigma_{n} / \sigma_{e}$, on est amené à faire certaines hypothèses sur la fonction de corrélation de l'interaction désorientatrice que nous ne savons pas justifier à ce stade sur le plan théorique. Pour élucider complètement le mécanisme de la relaxation du $\mathrm{Rb}$ en présence d'hélium, il apparaît que des mesures complémentaires sont nécessaires.

Abstract. - We present in this paper an experimental study of the relaxation in helium of $\mathrm{Rb}$ atoms polarized by optical pumping. Enriched isotopes ${ }^{87} \mathrm{Rb},{ }^{85} \mathrm{Rb},{ }^{4} \mathrm{He}$ and ${ }^{3} \mathrm{He}$ have been used. Different sources of systematic errors are discussed. The time evolution of $\left\langle S_{z}\right\rangle$ is found to involve two exponentials with time constants $\tau$ and $\tau^{\prime}$ whose variation versus helium pressure has been studied in the range 16 to 700 torrs. At low pressures, $\tau$ and $\tau^{\prime}$ are to be associated with the first and second diffusion modes of $\mathrm{Rb}$ atoins through the helium gas to the totally disorienting walls. This leads to the following values of the diffusion coefficient of $\mathrm{Rb}$ atoms in helium at atmospheric pressure and at $300 \circ \mathrm{K}: D_{0}\left(\mathrm{Rb}-{ }^{4} \mathrm{He}\right)=0.42 \pm 0.06 \mathrm{~cm}^{2} / \mathrm{s}$ which is found independent of the $\mathrm{Rb}$ isotope and $D_{0}\left(\mathrm{Rb}-{ }^{3} \mathrm{He}\right) / D_{0}\left(\mathrm{Rb}-{ }^{4} \mathrm{He}\right)=1.25 \pm 0.1$. The interpretation of these results is given.

At the opposite end of the explored pressure range, $\tau$ and $\tau^{\prime}$ are close to the time constants of the relaxation induced on $\left\langle S_{z}\right\rangle$ by gas collisions (a correction has to be done for substracting the effect induced by wall collisions). One thus gets the following cross-sections : $\sigma_{n}\left({ }^{87} \mathrm{Rb}-\mathrm{He}\right)$ $=0.47 \times 10^{-24} \mathrm{~cm}^{2}, \quad \sigma_{n}\left({ }^{85} \mathrm{Rb}-\mathrm{He}\right)=0.27 \times 10^{-24} \mathrm{~cm}^{2}$ for observable $\left\langle I_{z}\right\rangle$ and $\sigma_{e}(\mathrm{Rb}-\mathrm{He})$ $=8.2 \times 10^{-24} \mathrm{~cm}^{2}$ for observable $\left\langle Q_{e}\right\rangle$. Cross-sections relative to ${ }^{4} \mathrm{He}$ and ${ }^{3} \mathrm{He}$ are found the same; this is in agreement with the assumption according to which the relaxation of $\mathrm{Rb}$ atoms against rare gas atoms is due to the spin-orbit interaction. The effect of the nuclear spin of ${ }^{3} \mathrm{He}$ could not be detected, we conclude that it is smaller than was theoretically predicted. The ratio of the two cross-sections $\sigma_{n}$ relative to ${ }^{85} \mathrm{Rb}$ and ${ }^{87} \mathrm{Rb}$ is understood, as well as the equality of $\sigma_{e}$ for the two $\mathrm{Rb}$ isotopes. However to explain the value found for $\sigma_{n} / \sigma_{e}$, one is led to make assumptions about the correlation function of the disorienting interaction for which a theoretical justification is not available at the present time. Complementary measurements would be useful to elucidate more completely the relaxation of $\mathrm{Rb}$ in helium. 
Les expériences décrites dans ce travail ont été réalisées sur un mélange d'hélium et de vapeur de rubidium, contenu dans des cellules de verre pyrex (sans enduits de paraffine ou de silanes) dont les parois ont été volontairement contaminées par un miroir de $\mathrm{Rb}$. L'étude de la relaxation a été faite sur trois types de mélanges : ${ }^{87} \mathrm{Rb}-{ }^{3} \mathrm{He},{ }^{87} \mathrm{Rb}-{ }^{4} \mathrm{He},{ }^{85} \mathrm{Rb}-{ }^{4} \mathrm{He}$ dans le but d'élucider la nature de l'interaction désorientatrice.

Suivant une méthode déjà décrite [1], on procède en deux étapes : le système atomique est d'abord soumis au pompage optique et préparé dans un état initial différent de l'équilibre de Boltzmann; puis on interrompt le pompage et pendant le retour à l'équilibre on suit l'évolution au cours du temps d'une observable particulière, dans le cas présent $\left\langle S_{z}\right\rangle$. Ceci se fait en mesurant en fonction du temps la quantité de lumière absorbée sur un faisceau lumineux convenablement polarisé et assez peu intense pour ne pas perturber l'évolution sous le seul effet des collisions. Les mesures ont été faites avec des pressions d'hélium variant entre 16 et 670 torrs. Dans ces conditions, deux causes de relaxation sont importantes : les collisions du $\mathrm{Rb}$ contre la paroi après diffusion dans le gaz et les collisions du $\mathrm{Rb}$ contre l'hélium. L'évolution de $\left\langle S_{z}\right\rangle$ sous leur effet simultané a fait l'objet d'une étude théorique détaillée [2] à laquelle nous nous référons pour l'interprétation des mesures.

Nous indiquerons tout d'abord ( $(1)$ les problèmes expérimentaux particuliers que nous avons rencontrés pour l'étude de la relaxation en présence d'hélium. Ils sont liés essentiellement à la petitesse de la section efficace de désorientation $\mathrm{Rb}$-He, qui nécessite l'emploi de pressions d'hélium allant jusqu'à $1 \mathrm{~atm}$ en présence desquelles les raies d'absorption de la vapeur sont considérablement élargies et déplacées. Les résultats expérimentaux que nous décrirons ensuite $(\$ 2)$ permettent de déterminer les coefficients de diffusion $\mathrm{du} \mathrm{Rb}$ dans ${ }^{4} \mathrm{He}$ et ${ }^{3} \mathrm{He}$ ainsi que les sections efficaces de désorientation associées pour chaque isotope de $\mathrm{Rb}$ à deux observables qui évoluent différemment $\left(\left\langle I_{z}\right\rangle\right.$, l'orientation nucléaire, et $\left\langle Q_{e}\right\rangle=\left\langle S_{z}\right\rangle$ $\left.-\frac{2}{(2 I+1)^{2}-2}\left\langle I_{z}\right\rangle\right)$. Aucun effet lié au spin nucléaire de l'hélium n'a été observé sur ces sections efficaces. Nous achèverons $(\S 3)$ en comparant les valeurs des paramètres obtenus à celles qui ont été soit calculées théoriquement, soit mesurées par d'autres expérimentateurs.

1. Remarques sur les conditions expérimentales. 1. Remplissage Des Gellules. - Nous avons utilisé des cellules sphériques $(2 R=62 \mathrm{~mm})$ en verre pyrex. Chaque cellule est étuvée plusieurs heures à $450^{\circ} \mathrm{C}$; puis l'isotope de $\mathrm{Rb}\left({ }^{87} \mathrm{Rb}\right.$ enrichi à $99,2 \%$ ou ${ }^{85} \mathrm{Rb}$ enrichi à 99,6\%) est distillé à l'intérieur de la cellule de manière à former un mince miroir métallique sur toute la paroi, sauf sur les zones destinées à laisser passer les faisceaux lumineux. On introduit alors l'hélium ${ }^{4} \mathrm{He}$ (pureté $>99,999 \%$ ) ou ${ }^{3} \mathrm{He}$ (pureté isotopique $\approx 99,98 \%$ et pureté chimique $\approx 99,97 \%$, la principale impureté étant de l'hydrogène dont l'effet n'est pas décelable à cette concentration). La pression est mesurée à l'aide d'une jauge de Mac Leod. Chaque cellule est scellée et séparée du banc de pompage. Le miroir continu de $\mathrm{Rb}$ évolue par la suite et se rassemble en fines gouttelettes, mais la surface recouverte est suffisante pour que l'on puisse admettre que la pression $\mathrm{du} \mathrm{Rb}$ dans la cellule est à chaque température la tension de vapeur saturante.

2. Choix de LA Tension de VAPEUR DU Rb. Dans nos mesures, on doit déduire l'évolution de $\left\langle S_{z}\right\rangle$ de la variation $\Delta L_{\mathrm{A}}$ de la lumière absorbée $L_{\mathrm{A}}$ par la vapeur. Ceci impose donc que l'on opère dans des conditions telles que l'opacité équivalente $\bar{k} l$ [3] de la cellule soit nettement inférieure à 1 : il faut en effet que la variation de $L_{\mathrm{A}}$, lorsqu'on passe d'une vapeur très orientée à une vapeur désorientée :

$$
\Delta L_{\mathrm{A}}=\mathrm{e}^{-\bar{k}^{\prime} l}-\mathrm{e}^{-\bar{k} l}
$$

puisse s'écrire sous la forme $\bar{k} l-\bar{k}^{\prime} l$. Or nous avons opéré à des pressions de gaz très élevées (jusqu'à 600 torrs) pour lesquelles la raie d'absorption de la vapeur de $\mathrm{Rb}$ se trouve élargie et déplacée du fait des collisions. Pour une tension de vapeur du $\mathrm{Rb} p_{\mathrm{Rb}}$ fixée, on constate une grande diminution de la lumière absorbée quand la pression du gaz étranger $p$ croît, la raie d'absorption devenant plus large que la raie optique utilisée pour la détection. L'importance de cet effet est illustrée par la figure $1 \mathrm{a}$ où l'on a porté en fonction de $p$ la quantité $\bar{k} l$ (déduite de la mesure $\mathrm{du}$ rapport : lumière absorbée/lumière incidente $\left.=1-\mathrm{e}^{-\bar{k} l}\right)$. On peut noter que la courbe expérimentale présente aux environs de 400 torrs une brusque

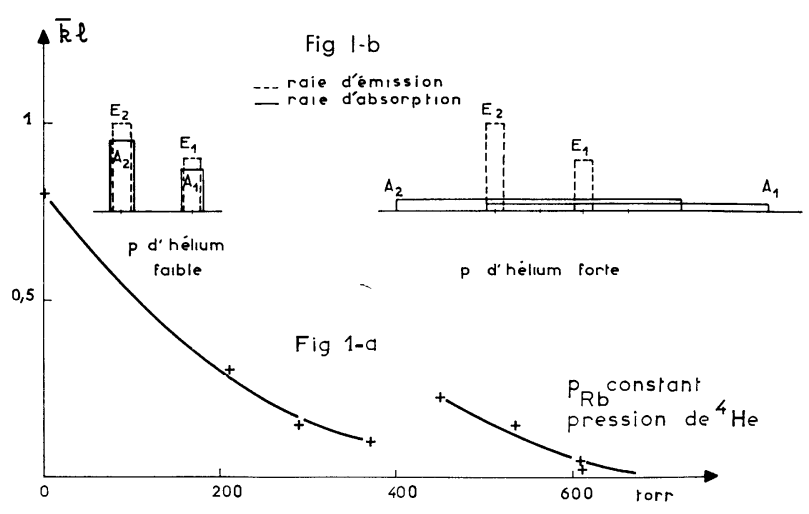

FIG. 1.

a) Variation de $\bar{k} l$ en fonction de la pression $p$ d'hélium à $p_{\mathrm{Rb}}$ constant $\left(1,5 \times 10^{-6}\right.$ torr). $\bar{k} l$ se déduit de la mesure du rapport : intensité de lumière $\mathrm{D}_{2}$ absorbée/intensité incidente. La source est une lampe de résonance.

b) Schéma du profil des raies d'émission et d'absorption dans les deux zones de pression extrêmes. 
discontinuité, à laquelle on a pu attribuer l'interprétation suivante [4] : aux pressions faibles, la raie d'absorption $\mathrm{D}_{2}$, comme la raie d'émission, se divise en deux composantes hyperfines parfaitement résolues (fig. $1 \mathrm{~b}$ ) : chaque raie d'absorption $\mathrm{A}_{1}$ (ou $\mathrm{A}_{2}$ ) ne recouvre qu'une seule raie d'émission $\mathrm{E}_{1}$ (ou $\mathrm{E}_{2}$ ). Quand la pression d'hélium augmente, une raie d'absorption telle que $A_{1}$ est élargie et déplacée de façon telle qu'elle peut empiéter sur la raie d'émission $E_{2}$ : certaines longueurs d'onde émises sur la raie $E_{1}$ peuvent être absorbées non seulement sur $A_{1}$ mais aussi sur $A_{2}$. D'où résulte le brusque changement de pente observé vers 400 torrs sur la courbe donnant la proportion de lumière absorbée. Au-delà de 400 torrs, quand $A_{1}$ et $A_{2}$ recouvrent à la fois $E_{1}$ et $E_{2}$, la variation de $\bar{k} l$ redevient monotone.

Comme conséquence des effets que nous venons de décrire, il est très difficile de réaliser des études de relaxation à des pressions d'hélium différentes en maintenant constante la tension de vapeur de $\mathrm{Rb}\left(p_{\mathrm{Rb}}\right)$. En effet, si la condition $\overline{k l}<1$ est satisfaite aux faibles pressions, la quantité de lumière absorbée aux fortes pressions devient si faible que le rapport signal sur bruit est catastrophique. Ainsi nous avons dû pour chaque pression d'hélium régler la tension de vapeur du $\mathrm{Rb}$ (c'est-à-dire la température $\theta$ de la cellule) de manière telle que l'absorption de la vapeur soit toujours sensiblement la même et telle que l'on ait $\bar{k} l \ll 1$ :

pour $p \lesssim 40$ torrs :

$$
p_{\mathrm{Rb}}=2,5 \times 10^{-7} \text { torr }\left(\theta=296{ }^{\circ} \mathrm{K}\right)
$$

pour $p \simeq 700$ torrs :

$$
p_{\mathrm{Rb}} \simeq 8 \times 10^{-6} \text { torr }(\theta=331 \mathrm{o} \mathrm{K}) .
$$

Dans ces conditions, le signal optique demeure facile à interpréter en fonction de la valeur moyenne de diverses observables relatives au système atomique. Gependant la détection de $\langle\mathbf{S}$. I $\rangle$ est, comme on peut le prévoir, très difficile, dès que l'élargissement des raies d'absorption optiques devient supérieur à la structure hyperfine. C'est du reste pourquoi nous n'avons pas encore étudié la relaxation de $\langle\mathbf{S} . \mathbf{I}\rangle$ pour le $\mathrm{Rb}$ en présence d'hélium.

La détection de $\left\langle S_{z}\right\rangle$ ne pose, elle, aucune difficulté lorsque $p_{\mathrm{Rb}}$ est judicieusement choisi à chaque pression d'hélium. Cependant on est conduit à se demander si les modifications apportées à la température $\theta$ du gaz et à la tension de vapeur du $\mathrm{Rb}$ ne peuvent pas affecter sa relaxation de manière appréciable. En fait, sur un intervalle $\Delta \theta$ aussi restreint, la fréquence des chocs $\mathrm{Rb}-\mathrm{He}\left(\propto \theta^{1 / 2}\right)$ et le coefficient de diffusion $\left(\propto \theta^{\mathbf{3} / 2}\right)$ sont affectés par la variation de $\theta$ au plus de 5 et $15 \%$. Chaque constante de temps mesurée peut du reste être corrigée si nécessaire puisque l'effet de la température peut être considéré comme approximativement connu. Nous admettrons que la section efficace moyenne de désorientation ne varie pas sur l'intervalle $\Delta \theta$ (ceci est justifié au $\S 3.2$ par l'équation (13)). Par contre, il reste l'effet des collisions d'échange $\mathrm{Rb}-\mathrm{Rb}$ qui pourrait ne pas demeurer sans importance (sur la relaxation de $\left\langle S_{z}\right\rangle$ ) aux tensions de vapeur élevées auxquelles on est amené à opérer. Pour répondre à cette question, les mesures de relaxation à une pression d'hélium fixée ont été répétées pour des valeurs de $p_{\mathrm{Rb}}$ variant dans un facteur de l'ordre de 2 : aucun effet attribuable à $p_{\mathrm{Rb}}$ n'a pu être observé sur les constantes de temps mesurées. En fait, il a déjà été montré que les collisions d'échange entre atomes identiques [5] ont un effet négligeable sur l'évolution de $\left\langle S_{z}\right\rangle$, mais il faut aussi envisager l'effet des collisions d'échange entre isotopes différents : bien que nous opérions sur des isotopes de $R b$ très purs, à forte tension de vapeur la fréquence des collisions ${ }^{85} \mathrm{Rb}-{ }^{87} \mathrm{Rb}$ n'est plus totalement négligeable. Elles ont pour effet de coupler entre elles les évolutions des observables des deux isotopes qui ont (en l'absence de couplage) des constantes de temps d'évolution propres très différentes. En fait, ceci altère beaucoup les constantes de temps de l'isotope peu concentré et très peu celles de l'isotope très concentré. Un calcul simple permet d'estimer que dans nos expériences l'erreur due à la présence de l'autre isotope est négligeable parce que nous avons opéré sur un seul isotope enrichi à plus de $99 \%$, mais il n'en serait plus de même pour une pureté isotopique 10 fois moindre.

3. Analyse des courbes de relaxation. - La courbe de relaxation de $\left\langle S_{z}\right\rangle$ est obtenue directement sur l'écran d'un oscilloscope où l'on peut la photographier ( fig. 2). En principe, dans le cas le plus général, cette courbe est la superposition de quatre exponentielles [2] :

$$
\begin{aligned}
\left\langle S_{z}\right\rangle=\gamma_{n}^{(1)} \mathrm{e}^{-t / \tau_{n}^{(1)}}+ & \gamma_{n}^{(2)} \mathrm{e}^{-t / \tau_{n}^{(2)}} \\
& +\gamma_{e}^{(1)} \mathrm{e}^{-t / \tau_{e}^{(1)}}+\gamma_{e}^{(2)} \mathrm{e}^{-t / \tau_{e}^{(2)}} .
\end{aligned}
$$

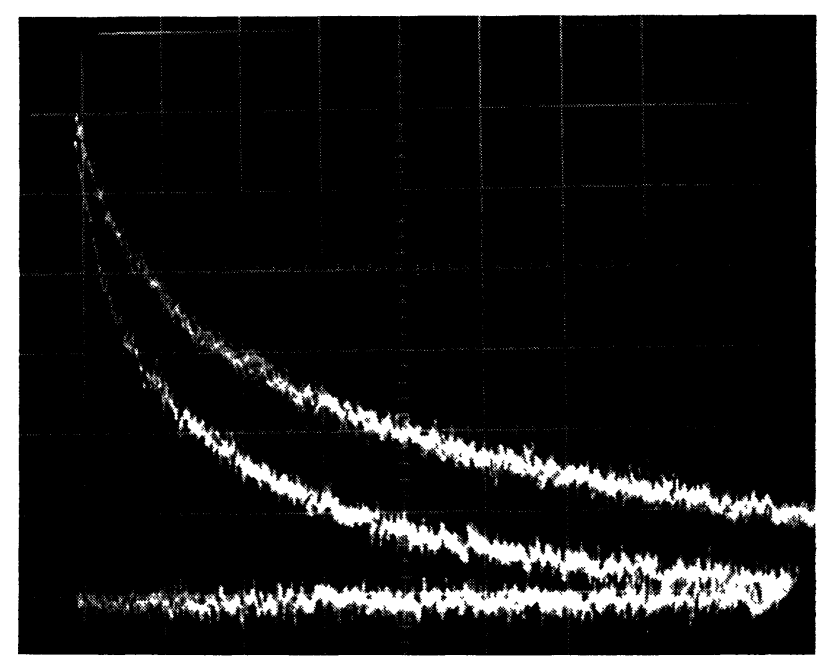

Fig. 2. - Exemple d'une courbe de relaxation de $\left\langle S_{z}\right\rangle$ photographiée sur un oscilloscope avec deux vitesses de balayage différentes; la trace horizontale représente l'asymptote. 
Chaque constante de temps $\tau_{i}^{\nu}$ caractérise l'évolution d'une observable donnée (indice $i=n$ pour $\left\langle I_{z}\right\rangle$, $i=e$ pour $\left.\left\langle Q_{e}\right\rangle=\left\langle S_{z}\right\rangle-\frac{2}{(2 I+1)^{2}-2}\left\langle I_{z}\right\rangle\right)$ sous l'effet simultané des chocs contre le gaz et sur la paroi après diffusion dans le gaz, suivant un mode temporel donné que désigne l'indice $v(1,2)$. Notre procédé graphique de dépouillement des courbes de relaxation ne nous permet pas d'obtenir les quatre constantes de temps mais seulement deux pseudoconstantes de temps $\tau$ et $\tau^{\prime}$ représentant en pratique la relaxation de $\left\langle S_{z}\right\rangle$ et telles que :

$$
\left\langle S_{z}\right\rangle=\gamma \mathrm{e}^{-t / \tau}+\gamma^{\prime} \mathrm{e}^{-t / \tau^{\prime}} \quad\left(\tau>\tau^{\prime}\right) \text {. }
$$

Nous déterminerons d'abord la constante de temps longue $\tau$ en amplifiant la partie de la courbe voisine de l'asymptote ( $t$ grand) : en coordonnée semi-logarithmique, cette portion de courbe est représentée par une droite de pente $-1 / \tau$. Ainsi déterminée, $\tau$ doit avoir une valeur voisine de la plus longue des constantes de temps d'évolution de $\left\langle S_{z}\right\rangle\left(\tau_{n}^{(1)}\right)$, d'autant plus que nous savons que parmi les quatre mentionnées plus haut c'est celle qui est affectée du poids le plus fort.

Une fois que nous connaissons $\tau$, nous considérons la courbe de relaxation complète (à partir de $t=0$ ). En coordonnées semi-logarithmiques, c'est une courbe $\mathscr{C}$, ayant pour asymptote une droite $\mathscr{D}$ de pente $-1 / \tau$. Nous retranchons les ordonnées des points de $\mathscr{C}$ et $\mathscr{D}$ ayant même abscisse : nous portons la différence en coordonnées semi-logarithmiques. Les points que nous obtenons ainsi ne sont alignés que si le second membre de l'équation (1) se réduit en pratique à la somme de deux termes soit parce que deux des coefficients sont très petits, soit parce que les constantes de temps prises deux à deux sont très voisines. Les circonstances où il en est ainsi sont connues [2] et ce n'est en fait que dans ce cas que la confrontation des résultats expérimentaux et théoriques est dépourvue d'ambiguité. Lorsque les points ne sont pas alignés, nous faisons passer une droite par les premiers points ( $t$ petit) de pente $-1 / \tau^{\prime}$. Il semble que nous obtenions par ce procédé la constante de temps la plus courte $\left(\tau_{e}^{(2)}\right)$. C'est du reste ce que l'on peut confirmer en analysant par ce procédé une courbe qui est la somme de quatre exponentielles connues. Mais l'incertitude avec laquelle cette constante de temps courte est évaluée dépend des grandeurs et des poids relatifs des diverses constantes de temps; elle est par conséquent très difficile à estimer.

2. Résultats expérimentaux. - Quelle que soit la pression d'hélium (entre 16 et 670 torrs), nous observons que la courbe de relaxation de $\left\langle S_{z}\right\rangle$ est caractérisée par deux constantes de temps $\tau$ et $\tau^{\prime}$ nettement distinctes. L'étude théorique $\mathrm{du}$ phénomène suggère une interprétation simple de $\tau$ et $\tau^{\prime}$ dans les deux cas limites suivants :

- Aux faibles pressions, les collisions sur la paroi, après diffusion dans le gaz, pour une paroi contaminée supposée totalement désorientante, représentent le processus de relaxation dominant pour toutes les observables $Q$. Ces dernières relaxent toutes de la même manière. Les deux constantes de temps qui apparaissent ne sont autres (quelle que soit $Q$ ) que $T^{(1)}$ et $T^{(2)}$ correspondant au temps de diffusion suivant les deux modes les plus bas.

- Aux fortes pressions, la relaxation par collisions sur les molécules du gaz l'emporte. Si l'interaction désorientatrice est de type magnétique faible, les deux constantes de temps qui apparaissent dans la relaxation de $\left\langle S_{z}\right\rangle$ doivent être $T_{e}^{\prime}$ et $T_{n}^{\prime}$ qui caractérisent la relaxation en phase gazeuse des deux observables différentes $\left\langle I_{z}\right\rangle$ et $\left\langle Q_{e}\right\rangle$.

Nous allons décrire successivement les résultats relatifs aux constantes de temps longue $\tau$ et courte $\tau^{\prime}$.

1. Constante de temps longue $\tau$. - La figure 3 représente les résultats relatifs à $1 / \tau$ en fonction de $p$

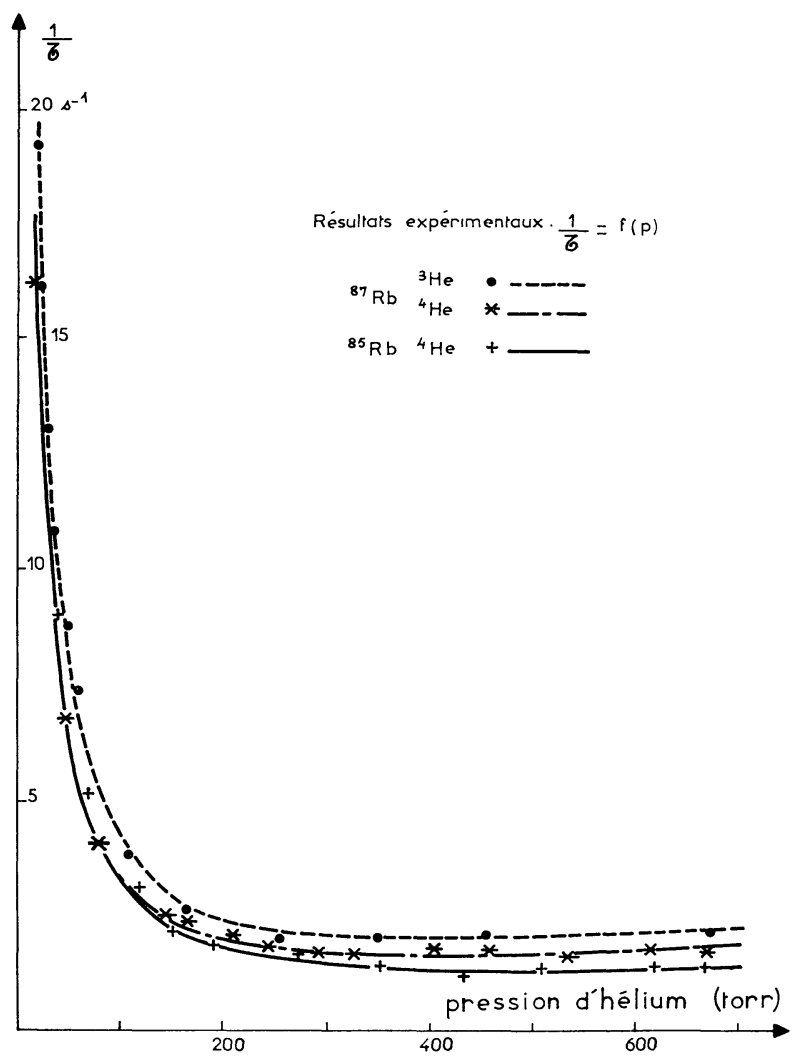

FIG. 3. - Résultats expérimentaux : 1'inverse de la constante de temps longue $\tau$ en fonction de la pression $p$ d'hélium.

dans les trois cas étudiés : ${ }^{87} \mathrm{Rb}-{ }^{3} \mathrm{He} ;{ }^{87} \mathrm{Rb}-{ }^{4} \mathrm{He} ;{ }^{85} \mathrm{Rb}-$ ${ }^{4} \mathrm{He}$.

On constate que les courbes expérimentales ont toutes les trois l'allure de la courbe théorique [2] donnant la variation de $1 / \tau_{n}^{(1)}$ avec $p$ dans le cas où les parois sont totalement désorientantes. Rappelons 
que $\tau_{n}^{(1)}$ est la constante de temps associée au premier mode de diffusion pour une observable dont la relaxation sur le gaz seul est caractérisée par une constante de temps unique $T_{n}^{\prime}$ :

$$
\frac{1}{\tau_{n}^{(1)}}=\frac{1}{T^{(1)}}+\frac{1}{T_{n}^{\prime}}
$$

Aux faibles pressions d'hélium $(p<150$ torrs), on observe essentiellement le premier terme qui traduit la relaxation sur la paroi après diffusion dans le gaz selon le premier mode : $1 / \tau_{n}^{(1)} \approx 1 / T^{(1)}\left(T^{(1)}\right.$ ne dépend pas de l'observable lorsque la désorientation produite par les chocs sur la paroi est totale). On voit que du côté des fortes pressions on commence tout juste à observer entre 400 et 670 torrs l'augmentation de $1 / \tau$ avec $p$ liée à $1 / T_{n}^{\prime}$. Il faudrait opérer à une pression d'au moins deux atmosphères pour que la relaxation sur le gaz soit vraiment le processus dominant.

Lorsqu'on étudie en détail la forme des courbes expérimentales précédentes, on trouve que chacune d'entre elles se déduit avec une très bonne approximation d'une hyperbole $\mathrm{G}$ et d'une droite $\mathrm{C}^{\prime}$ en faisant la somme des ordonnées des points de $\mathrm{C}$ et $\mathrm{C}^{\prime}$ ayant même abscisse, comme cela est le cas pour la courbe théorique $\Gamma$ relative à $1 / \tau_{n}^{(1)}$. On peut en conclure que, dans toute la zone des pressions explorées, la constante de temps longue $\tau$ que nous mesurons est la quantité $\tau_{n}^{(1)}$ définie par la relation (3), puisque nous savions déjà que $\tau$ représente $T_{n}^{(1)}$ en pression très faible et $T_{n}^{\prime}$ en pression très forte.

Dans chacun des trois mélanges étudiés, on peut déterminer l'hyperbole $\mathrm{G}$ et la droite $\mathrm{C}^{\prime}$ de manière que la courbe théorique $\Gamma$ (« somme » de $\mathrm{G}_{\text {et }} \mathrm{C}^{\prime}$ ) passe au mieux par l'ensemble des points expérimentaux. Les courbes théoriques $\left(\mathrm{C}, \mathrm{G}^{\prime}, \Gamma\right)$ et les points expérimentaux sont représentés sur les figures 4 et 5 . De l'équation de $\mathrm{C}$ on peut déduire le coefficient de diffusion $D$ et de la pente de $\mathrm{C}^{\prime}$ la section efficace de désorientation $\sigma_{n}$ relative à l'observable $\left\langle I_{z}\right\rangle$ si l'interaction désorientatrice en phase gazeuse est de type magnétique.

a) Détermination des coefficients de diffusion. - On remarque sur la figure 3 que les courbes expérimentales concernant ${ }^{4} \mathrm{He}$ et les deux isotopes de $\mathrm{Rb}$ sont confondues; ceci signifie que le coefficient de diffusion de ${ }^{87} \mathrm{Rb}$ et ${ }^{85} \mathrm{Rb}$ dans l'hélium est pratiquement le même. Par contre, la courbe expérimentale relative à ${ }^{3} \mathrm{He}$ se place nettement au-dessus de la précédente : le coefficient de diffusion de $\mathrm{Rb}$ dans ${ }^{3} \mathrm{He}$ est donc plus grand que dans ${ }^{4} \mathrm{He}$ comme on devait s'y attendre. Toutes les mesures utiles pour la détermination du coefficient de diffusion $(p \lesssim 200$ torrs $)$ ont été faites à des températures voisines de $300 \mathrm{oK}\left(\Delta \theta<10^{\circ}\right)$, aussi nous n'avons pas fait de correction de température sur les valeurs mesurées. Les équations des courbes $\mathrm{C}$ tracées sur la figure 4 sont indiquées dans le tableau I, ainsi que les valeurs des coefficients de diffusion $D_{0}$ (à $300 \mathrm{oK}$

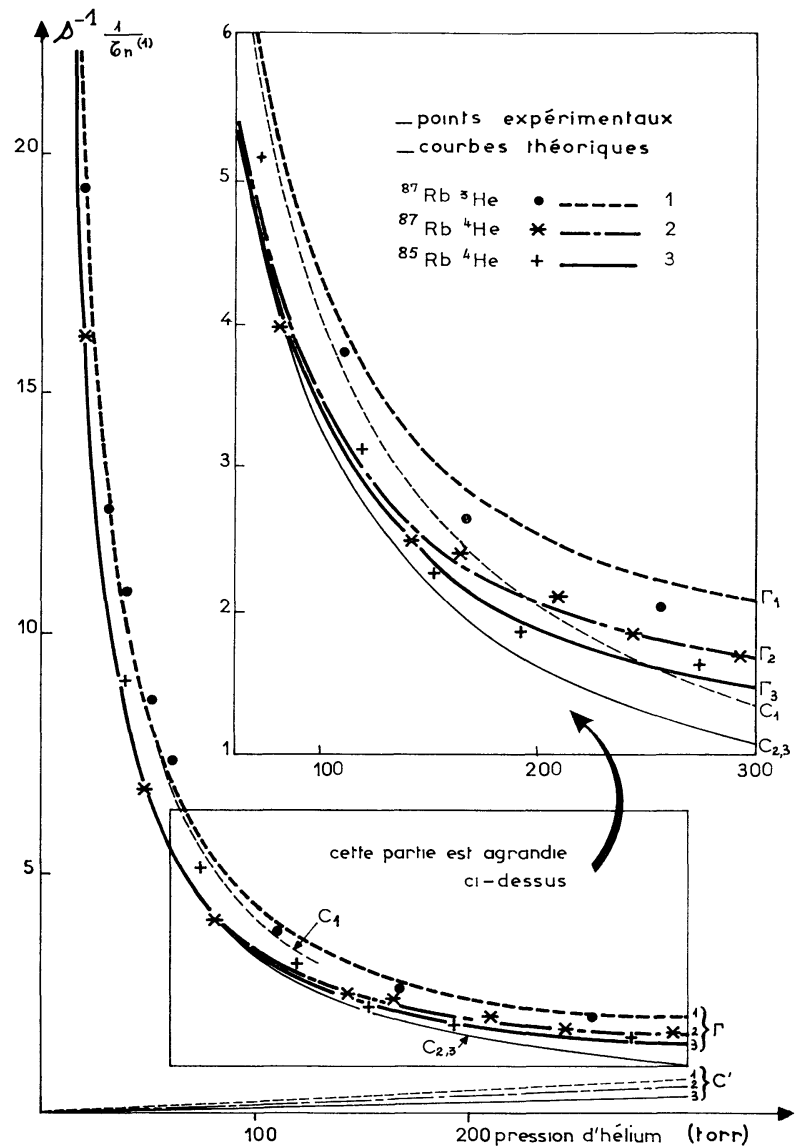

FIG. 4. - Détermination des coefficients de diffusion : points expérimentaux $1 / \tau$ et courbes théoriques $\Gamma$ " sommes " de $\mathrm{C}$ et $\mathrm{C}^{\prime}$ donnant $1 / \tau_{n}^{(1)}=1 / T^{(1)}+1 / T_{n}^{\prime}$ dans la zone de pression où la désorientation est produite essentiellement par les collisions sur la paroi après diffusion dans le gaz $\left(1 / T_{n}^{\prime} \ll 1 / T^{(1)}\right)$.

\section{TABLEAU I}

\section{RÉSULTATS EXPÉRIMENTAUX}

RELATIFS A LA CONSTANTE DE TEMPS LONGUE ET A LA DÉSORIENTATION PAR GHOCS SUR LA PAROI APRÈS DIFFUSION

( $p$ représente la pression d'hélium mesurée en torrs à $300 \mathrm{oK}$ )

\begin{tabular}{|c|c|c|}
\hline & $\begin{array}{c}1 / T^{(1)}\left(\mathrm{s}^{-1}\right) \\
-\end{array}$ & $\begin{array}{c}D_{0}\left(\mathrm{~cm}^{2} \mathrm{~s}^{-1}\right) \\
\text { à } 300 \mathrm{oK} \\
-\end{array}$ \\
\hline${ }^{87} \mathrm{Rb}-{ }^{3} \mathrm{He}$ & $450 p^{-1}$ & $0,53 \pm 0,08$ \\
\hline 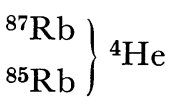 & $360 p^{-1}$ & $0,42 \pm 0,06$ \\
\hline
\end{tabular}


et à la pression atmosphérique $p_{0}$ ) obtenues à l'aide de la relation :

$$
1 / T^{(1)}=D_{0} \frac{p_{0}}{p} \frac{\pi^{2}}{R^{2}}
$$

valable pour une cellule sphérique de rayon $R$.

On obtient en particulier :

$$
\frac{D_{0}\left(\mathrm{Rb}-{ }^{3} \mathrm{He}\right)}{D_{0}\left(\mathrm{Rb}-{ }^{4} \mathrm{He}\right)}=1,25 \pm 0,1 .
$$

L'incertitude est en valeur relative plus faible pour ce rapport que pour la valeur absolue de chacun des coefficients de diffusion. Étant donné que ces deux coefficients sont voisins, on peut en effet raisonnablement supposer que les erreurs systématiques sont les mêmes et s'éliminent sur le rapport.

b) Détermination de la section efficace de désorientation $\sigma_{n}$. - On remarque sur la figure 3 que les points expérimentaux relatifs à ${ }^{4} \mathrm{He}$ et aux deux isotopes de $\mathrm{Rb}$ se placent sur deux courbes qui deviennent nettement distinctes à des pressions supérieures à 300 torrs. Les temps de relaxation les plus longs correspondent à ${ }^{85} \mathrm{Rb}$. Ceci signifie que la section efficace $\sigma_{n}$ dépend du spin nucléaire : c'est bien qualitativement ce que l'on attend si l'interaction désorientatrice en phase gazeuse est de type magnétique faible. On voit aussi sur la même figure que l'écart entre les courbes expérimentales ${ }^{87} \mathrm{Rb}-{ }^{3} \mathrm{He}$ et ${ }^{87} \mathrm{Rb}-{ }^{4} \mathrm{He}$ est pratiquement indépendant de la pression entre 300 et 670 torrs. Aux fortes pressions, cet écart ne s'explique plus par la différence entre les coefficients de diffusion, il signifie que les temps de relaxation $T_{n}^{\prime}$ sur le gaz sont différents dans ${ }^{4} \mathrm{He}$ et ${ }^{3} \mathrm{He}$ à pression égale. Comme la fréquence des collisions que subit le $\mathrm{Rb}$ n'est pas la même dans les deux cas $\left({ }^{3} \mathrm{He}\right.$ plus léger produit des collisions plus fréquentes), ce fait n'implique pas nécessairement que les sections efficaces $\mathrm{Rb}-{ }^{4} \mathrm{He}$ et $\mathrm{Rb}-{ }^{3} \mathrm{He}$ soient différentes.

Aux points expérimentaux représentés sur la figure 5 , nous devons appliquer une correction de température qui n'excède jamais $5 \%$. Après moyenne des résultats, nous obtenons les valeurs de $1 / T_{n}^{\prime}$ qui sont portées dans le tableau II pour les trois couples étudiés. On peut

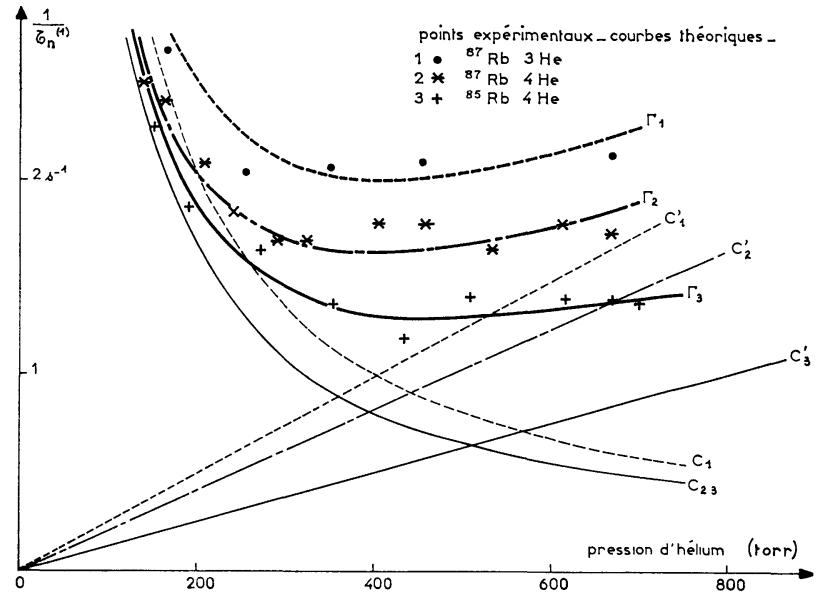

FIG. 5. - Détermination des sections efficaces $\sigma_{n}$ : points expérimentaux $1 / \tau$ et courbes théoriques $\Gamma$ dans la zone de pression où les chocs contre le gaz deviennent importants.

en déduire les sections efficaces de désorientation en utilisant la relation :

$$
\sigma_{n}=\left(T_{n}^{\prime} \bar{V}_{r} n\right)^{-1}
$$

$\left(\bar{V}_{r}\right.$ vitesse relative moyenne des atomes entrant en collision et $n$ nombre d'atomes par $\mathrm{cm}^{3}$; les valeurs numériques de la quantité $\bar{V}_{r} n$ sont aussi indiquées dans le tableau II).

En ce qui concerne les rapports entre les sections efficaces $\sigma_{n}$, nous obtenons les valeurs :

$$
\begin{aligned}
& \frac{\sigma_{n}\left({ }^{85} \mathrm{Rb}-{ }^{4} \mathrm{He}\right)}{\sigma_{n}\left({ }^{87} \mathrm{Rb}-{ }^{3} \mathrm{He}\right)}=1,73 \pm 0,5 \\
& \frac{\sigma_{n}\left({ }^{87} \mathrm{Rb}-{ }^{3} \mathrm{He}\right)}{\sigma_{n}\left({ }^{87} \mathrm{Rb}-{ }^{4} \mathrm{He}\right)}=1,03 \pm 0,15 .
\end{aligned}
$$

L'erreur relative est plus grande sur $\sigma_{n}$ que sur $D_{0}$, elle peut paraître à première vue surévaluée étant donné la dispersion assez faible des points expérimentaux portés sur la figure 3 . La raison est en fait la suivante : dans le domaine de pression où sont faites les mesures, la diffusion vers la paroi a encore une

\section{TABLEAU II}

RÉSUltats EXPÉRIMENTAUX RELATIFS A LA CONSTANTE DE TEMPS LONGUE ET A LA CONTRIBUTION DES GOLLisions Rb-He

( $p$ représente la pression d'hélium mesurée en torrs à $300 \mathrm{oK}$ )

$\begin{array}{cccc} & 1 / T_{n}^{\prime}\left(\mathrm{s}^{-1}\right) & \bar{V}_{r} n\left(\mathrm{~cm}^{-2} \mathrm{~s}^{-1}\right) & \sigma_{n}\left(\mathrm{~cm}^{2}\right) \\ & - & - & - \\ { }^{87} \mathrm{Rb}-{ }^{3} \mathrm{He} & 2,25 p 10^{-3} & 4,74 p 10^{21} & 0,47 \pm 0,15 \times 10^{-24} \\ { }^{87} \mathrm{Rb}-{ }^{4} \mathrm{He} & 1,9 p 10^{-3} & 4,13 p 10^{21} & 0,46 \pm 0,15 \times 10^{-24} \\ { }^{85} \mathrm{Rb}-{ }^{4} \mathrm{He} & 1,1 p 10^{-3} & 4,14 p 10^{21} & 0,27 \pm 0,1 \times 10^{-24}\end{array}$


importance fort appréciable et les collisions sur le gaz sont seulement responsables de 50 à $75 \%$ de la valeur de $1 / \tau$ mesurée sur ${ }^{87} \mathrm{Rb}$ (40 à $65 \%$ dans le cas de ${ }^{85} \mathrm{Rb}$ ). On peut alors craindre d'introduire des erreurs systématiques difficiles à évaluer exactement. Il se trouve que les temps de relaxation mesurés sur $1 \mathrm{e}^{87} \mathrm{Rb}$ mélangé à ${ }^{4} \mathrm{He}$ ou ${ }^{3} \mathrm{He}$ sont très voisins, donc il semble raisonnable d'admettre que les erreurs systématiques commises sur $\sigma_{n}\left({ }^{87} \mathrm{Rb}-{ }^{3} \mathrm{He}\right)$ et $\sigma_{n}\left({ }^{87} \mathrm{Rb}-{ }^{4} \mathrm{He}\right)$ s'éliminent sur leur rapport. Par contre, ceci n'est plus vrai pour le rapport des $\sigma_{n}$ relatifs aux deux isotopes de $\mathrm{Rb}$ qui est de l'ordre de 2.

Parmi les causes possibles d'erreurs systématiques, celle qui nous semble la plus importante résulte de ce que $\left\langle S_{z}\right\rangle$ évolue en principe avec quatre constantes de temps distinctes tant que la relaxation par collisions sur le gaz n'est pas le processus dominant. Comme nous l'avons expliqué précédemment ( $\$ 1.3$ ), nous savons seulement que la valeur $\tau$ mesurée est voisine de $\tau_{n}^{(1)}$. Nous avons cherché à évaluer l'erreur correspondante de la manière suivante. D'abord, nous traçons pour plusieurs valeurs de $p$ la courbe théorique qui décrit en principe l'évolution de $\left\langle S_{z}\right\rangle$. Gette courbe est définie par l'équation (1), les paramètres $\gamma_{i}^{(v)}$ et $\tau_{i}^{(v)}$ sont évalués à l'aide des expressions théoriques de la référence [2] et des valeurs des paramètres $D_{0}, \sigma_{n}, \sigma_{e}$ déduits de nos mesures (on trouve que dans la zone de pression explorée $\tau_{n}^{(1)} / \tau_{n}^{(2)}$ varie entre 1,8 et 2,5 , $\gamma_{n}^{(1)} / \gamma_{n}^{(2)}>1$ et que $\tau_{e}^{(2)}$ est très voisin de $\left.\tau_{e}^{(1)}\right)$. Ensuite, nous analysons les courbes théoriques ainsi calculées comme nous le faisons pour les courbes expérimentales. Nous obtenons une constante de temps longue $\tau$ plus courte que $\tau_{n}^{(1)}$ à cause de la présence de $\tau_{n}^{(2)}$, mais la différence entre $1 / \tau$ mesuré et $1 / \tau_{n}^{(1)}$ demeure inférieure à $10 \%$.

2. Constante De temps gourte $\tau^{\prime}$. - Les résultats expérimentaux concernant la constante de temps courte sont représentés figure 6 . Cette fois les points relatifs aux trois cas étudiés se situent sur une courbe unique qui présente un minimum. Nous distinguerons trois zones de pression.

Dans la zone des faibles pressions ( $p<100$ torrs), la quantité $1 / \tau^{\prime}$ qui décroît rapidement quand $p$ croît peut s'interpréter comme étant associée au second mode de diffusion vers la paroi $1 / T^{(2)} \approx 1 / \tau_{n}^{(2)} \approx 1 / \tau_{e}^{(2)}$. En effet, nous avons tracé, à partir de l'hyperbole de diffusion $\mathrm{C}^{(1)}$ représentant le premier mode, l'hyperbole de diffusion $\mathrm{C}^{(2)}$ représentant le second mode :

$$
1 / T^{(2)}=4 / T^{(1)} \text {. }
$$

Les courbes $\mathrm{C}_{2-3}^{(2)}$ associées aux couples ${ }^{87} \mathrm{Rb}-{ }^{4} \mathrm{He}$ et ${ }^{85} \mathrm{Rb}-{ }^{4} \mathrm{He}$ sont confondues et voisines de la courbe $\mathrm{C}_{1}^{(2)}$ associée au couple ${ }^{87} \mathrm{Rb}-{ }^{3} \mathrm{He}$. A cause de l'incertitude des mesures (la détermination de $\tau^{\prime}$ est moins précise que celle de $\tau$ ), on constate que tous les points expérimentaux, qu'ils soient relatifs à ${ }^{4} \mathrm{He}$ ou à ${ }^{3} \mathrm{He}$, se dispersent autour des deux courbes théoriques $\mathrm{C}_{2-3}^{(2)}$ et $\mathrm{C}_{1}^{(2)}$.

LE JOURNAL DE PHYSIQUe. - T. 30. N $^{\text {os }}$ 8-9. AOUT-SEPTEMBRE 1969

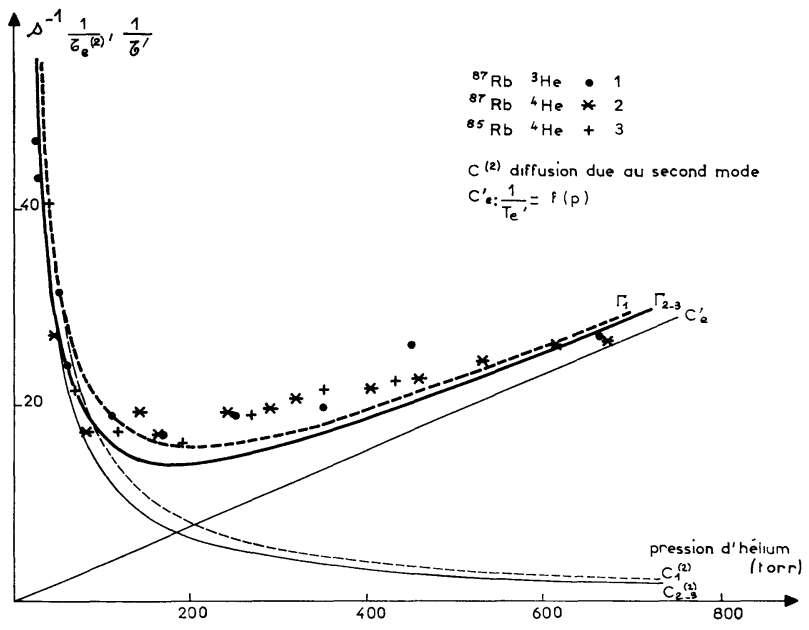

Fig. 6. - L'inverse de la constante de temps courte associée à la relaxation de $\left\langle S_{z}\right\rangle$ en fonction de la pression $p$ d'hélium : points expérimentaux $1 / \tau^{\prime}$ et courbes théoriques $\Gamma$ " somme " de $\mathrm{C}^{(2)}$ et $\mathrm{C}^{\prime}$ donnant $1 / \tau_{e}^{(2)}=1 / T^{(2)}+1 / T_{e}^{\prime}$.

Signalons que dans cette zone les poids $\gamma_{1}, \gamma_{2}$ des constantes de temps $T^{(1)}, T^{(2)}$ sont tels que $\gamma_{2} / \gamma_{1} \sim 0,25$ pour un faisceau pompant de forte intensité $\left(T_{p}<T^{(2)}\right)$ éclairant presque uniformément tout le volume de la cellule. Ce résultat est en bon accord avec ce que prévoit l'étude théorique [2].

Dans la zone des fortes pressions ( $p>400$ torrs), $1 / \tau^{\prime}$ qui est passé par un minimum vers 180 torrs croît avec $p$; les valeurs mesurées sont grandes devant les ordonnées des courbes $\mathrm{G}^{(1)}$ et $\mathrm{C}^{(2)}$, c'est-à-dire que la relaxation sur la paroi après diffusion dans le gaz selon le premier ou le second mode est négligeable : $\tau^{\prime}$ peut s'interpréter comme la constante de temps associée à la relaxation par collisions sur le gaz d'une observable particulière; on s'attend à ce que cette observable soit $\left\langle Q_{e}\right\rangle$ si l'interaction désorientatrice est de type magnétique. On mesure la même constante de temps sur les deux isotopes de $\mathrm{Rb}$ pour une même valeur de $p$. C'est bien ce qu'on prévoit pour une interaction aléatoire de type magnétique.

Comme le montre la figure 7 , le poids de la constante de temps $\tau^{\prime}$ dépend beaucoup dans cette zone de l'intensité pompante : en réduisant cette dernière dans un facteur 4 , on a fait varier de 0,8 à 0,38 le rapport $\gamma_{e} / \gamma_{n}$ qui s'interprète comme étant égal à la quantité $\left\langle Q_{e}\right\rangle_{0} /\left\langle I_{z}\right\rangle_{0}$ (l'indice 0 désignant l'état initial à partir duquel s'effectue la relaxation).

Dans la zone de pression intermédiaire où $1 / \tau^{\prime}$ passe par un minimum, l'interprétation des mesures est tres délicate puisqu'en principe l'évolution de $\left\langle S_{z}\right\rangle$ est caractérisée par quatre constantes de temps distinctes. Cependant on constate dans ce domaine de pression comme dans les deux autres que les points expérimentaux se placent avec une assez bonne approximation sur la courbe $\Gamma$ 


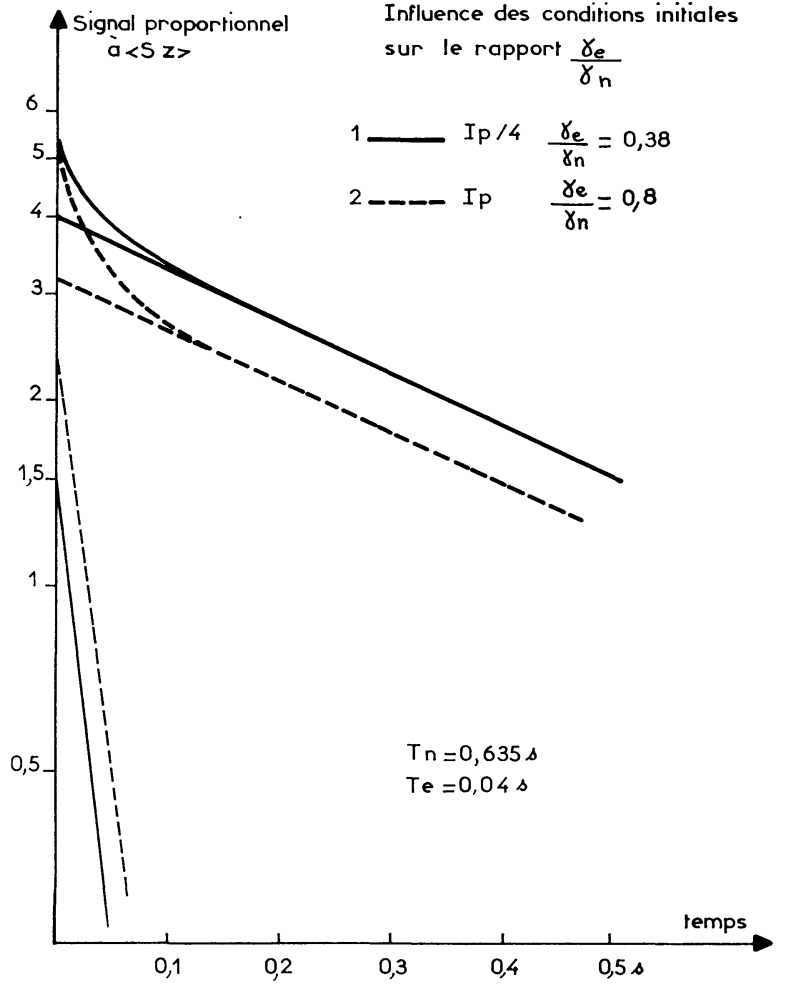

FIG. 7. - Influence de l'intensité pompante $I_{p}$ sur le poids relatif $\gamma_{e} / \gamma_{n}$ des deux constantes de temps associées à l'évolution de $\left\langle S_{z}\right\rangle$ (la figure représente les courbes de relaxation de $\left\langle S_{z}\right\rangle$ pour $I_{p}$ et $I_{p} / 4$ en coordonnées semi-logarithmiques et leur dépouillement à l'aide de deux constantes de temps).

déduite d'une hyperbole $\mathrm{C}^{(2)}$ (représentant la variation de $1 / T^{(2)}$ avec $p$ ) et d'une droite $\mathrm{C}_{e}^{\prime}$ (représentant la variation de $1 / T_{e}^{\prime}$ avec $p$ ) en faisant la somme des ordonnées des points de $\mathrm{C}^{(2)}$ et $\mathrm{C}_{e}^{\prime}$ ayant même abscisse. Ceci veut dire que même dans le domaine de pression intermédiaire la constante de temps courte mesurée peut s'interpréter comme étant $\tau_{e}^{(2)}$. Il est probable que cela provient du procédé graphique utilisé pour analyser les courbes expérimentales : comme nous l'avons fait remarquer $(\S 1.3)$, ce procédé tend à donner la plus longue et la plus courte des quatre constantes de temps.

De la pente de la droite $\mathrm{C}_{e}^{\prime}$ :

$$
1 / T_{e}^{\prime}=3,6 p 10^{-2} \mathrm{~s}^{-1} \quad(p \text { en torrs })
$$

nous déduisons la section efficace de désorientation :

$$
\sigma_{e}=8,2 \times 10^{-24} \mathrm{~cm}^{2}
$$

que nous trouvons bien indépendante de l'isotope.

Pour les rapports entre sections efficaces, nous obtenons :

$$
\begin{array}{ll}
\sigma_{e} / \sigma_{n}=18 \pm 5 & \text { pour }{ }^{87} \mathrm{Rb} \\
\sigma_{e} / \sigma_{n}=30,5 \pm 9 & \text { pour }{ }^{85} \mathrm{Rb} .
\end{array}
$$

3. Discussion des résultats. - 1 . Coefficient DE DIFFusion. - Le phénomène de diffusion étudié ici correspond au cas d'atomes très lourds (atomes $1=\mathrm{Rb}$ ) diffusant dans un gaz très léger (atomes $2=\mathrm{He}$ ) et son caractère essentiel est la persistance de la vitesse de l'atome lourd au cours d'un grand nombre de chocs sur l'atome léger [6]. On peut ainsi comprendre pourquoi le coefficient de diffusion mesuré est bien plus grand (environ 20 fois) que celui qu'on calcule en appliquant la formule élémentaire $D=\frac{\hbar \bar{v}}{3} \quad(l$ libre parcours moyen, $\bar{v}$ vitesse moyenne du $\mathrm{Rb}$ ).

Dans le cadre de la théorie cinétique des gaz dilués, le coefficient de diffusion d'un mélange binaire est donné en première approximation par l'expression (7) :

$\mathscr{D}_{12}\left(p_{0}\right)=2,63 \times 10^{-3} \frac{\left[\theta^{3}\left(M_{1}+M_{2} / 2 M_{1} M_{2}\right)\right]^{1 / 2}}{\sigma_{12}^{2} \xi_{12}}$

$\left(\mathscr{D}_{12}\right.$ en $\mathrm{cm}^{2} \mathrm{~s}^{-1}, \theta$ en $\mathrm{oK}, \sigma_{12}$ en $\AA, M_{1}, M_{2}$ masses moléculaires). Les quantités qui apparaissent au dénominateur de cette expression sont caractéristiques du potentiel d'interaction hétéronucléaire $V_{12}(r): \sigma_{12}$ représente la plus faible distance à laquelle $V_{12}(r)=0$; $\xi_{12}$ est un nombre sans dimension qui dépend de la forme du potentiel et qui est susceptible de varier lentement avec la température. Il est défini de manière à être égal à 1 dans le cas d'un potentiel de sphères dures $\left(V_{12}(r) \infty\right.$ pour $r \leqslant \sigma_{12}, 0$ pour $\left.r>\sigma_{12}\right)$ et il est tabulé dans la référence [7] pour un potentiel $V_{12}(r)$ représenté par un Lennard-Jones 12-6. Sa valeur indique donc à quel degré le gaz s'écarte d'un gaz de sphères dures du point de vue de la diffusion.

Nous ne disposons d'aucune donnée sur le potentiel interatomique Rb-He. Cependant un ordre de grandeur pour $\sigma_{12}$ peut se déduire de la loi d'intercombinaison :

$$
\sigma_{12}=\frac{1}{2}\left(\sigma_{\mathrm{He}}+\sigma_{\mathrm{Rb}}\right)
$$

en utilisant $\sigma_{\mathrm{He}}=2,63 \AA$ [7] et en déduisant

$$
\sigma_{\mathrm{Rb}}=4,7 \AA
$$

des potentiels $\mathrm{Rb}$-gaz rares [8], toujours à l'aide de la loi d'intercombinaison. On trouve $\sigma_{12}=3,6 \AA$. En reportant cette valeur dans l'expression (12) et en prenant pour $\mathscr{D}_{12}\left(p_{0}\right)$ la valeur expérimentale $D_{0}$ mesurée à $300 \mathrm{~K}$, on obtient le facteur de forme :

$$
\xi_{12}=0,83 \text { à partir de } D_{0}\left({ }^{87} \mathrm{Rb}-{ }^{3} \mathrm{He}\right)
$$

et

$$
\xi_{12}=0,90 \text { à partir de } D_{0}\left({ }^{87} \mathrm{Rb}-{ }^{4} \mathrm{He}\right) \text {. }
$$

La différence $1-\xi_{12} \approx 0,14$ nous paraît significative. Notons qu'elle a le signe et l'ordre de grandeur de celle $(0,24)$ qu'on calcule en représentant $V_{12}(r)$ par un Lennard-Jones ayant les mêmes caractéristiques que pour la paire $\mathrm{He}-\mathrm{Kr}$ ( $\mathrm{Kr}$ et $\mathrm{Rb}$ ne différant que par la présence d'un seul électron $s$, leurs interactions électrostatiques avec l'hélium sont du même ordre de grandeur). 
En ce qui concerne le rapport

$$
D_{0}\left({ }^{87} \mathrm{Rb}-{ }^{3} \mathrm{He}\right) / D_{0}\left({ }^{87} \mathrm{Rb}-{ }^{4} \mathrm{He}\right)
$$

la valeur théorique 1,15 déduite de l'expression (12) est compatible avec la valeur expérimentale $1,25 \pm 0,1$.

Remarquons enfin que la variation du coefficient de diffusion en $\theta^{3 / 2}$ n'est valable qu'en première approximation puisque $\xi_{12}$ peut dépendre de $\theta$. L'étude de $D_{0}$ avec la température serait en fait un bon moyen d'obtenir le potentiel hétéronucléaire à partir de propriétés macroscopiques du gaz, étant donné que les forces entre atomes identiques n'affectent pratiquement pas la valeur de $D_{0}$.

2. Segtions efficages de DÉsorientation. - Pour les gaz rares qui n'ont pas de spin nucléaire, on s'attend à ce que la relaxation du $\mathrm{Rb}$ soit causée par l'interaction « spin-orbite» [9]. Dans le cas où le gaz rare possède un spin nucléaire $\mathbf{K}$, il faut aussi tenir compte de l'interaction magnétique entre $\mathbf{S}$ et $\mathbf{K}$ qui fait intervenir l'interaction entre les dipôles magnétiques ponctuels, et surtout un terme de contact résultant des forces d'échange entre l'électron de valence de $\mathrm{Rb}$ et les électrons de l'hélium [10], [11]. Pour la paire $\mathrm{Rb}-\mathrm{He}$, Herman a évalué un ordre de grandeur des sections efficaces associées à l'interaction « spin-orbite» $\left(3,8 \times 10^{-24} \mathrm{~cm}^{2}\right.$ [9]) et à l'interaction magnétique $\mathbf{S} . \mathbf{K}\left(1,1 \times 10^{-24} \mathrm{~cm}^{2}[10]\right)$. Cette dernière ne peut être présente que pour l'isotope ${ }^{3} \mathrm{He}$, et si sa contribution est aussi grande quele prévoit Herman, on doit obtenir : $\sigma_{n}\left({ }^{87} \mathrm{Rb}-{ }^{3} \mathrm{He}\right) / \sigma_{n}\left({ }^{87} \mathrm{Rb}-{ }^{4} \mathrm{He}\right)=1,3$. En fait, nous observons pour ce rapport la valeur 1 . Nous pouvons en conclure que l'interaction désorientatrice dépend peu du moment magnétique nucléaire de l'hélium, moins qu'il n'avait été prévu théoriquement. D’autre part, notons que la différence de masse entre ${ }^{3} \mathrm{He}$ et ${ }^{4} \mathrm{He}$ entraîne une différence entre la durée moyenne $\tau_{\text {coll }}$ des collisions $\mathrm{Rb}-{ }^{4} \mathrm{He}$ et $\mathrm{Rb}-{ }^{3} \mathrm{He}$; puisque nous mesurons la même section efficace, c'est-à-dire la même probabilité de désorientation par collision $\overline{\left|\mathscr{H}_{1}\right|^{2}} \hbar^{-2} \tau_{\text {coll }}^{2}$, il semble que la force de l'interaction
$\left|\mathscr{H}_{1}\right|^{2}$ dépend elle-même de la vitesse $V_{r}$. Ceci est tout à fait compatible avec l'hypothèse selon laquelle l'interaction désorientatrice est l'interaction spinorbite pour la paire $\mathrm{Rb}-\mathrm{He}$. Gette interaction résulte en effet de l'apparition pendant la durée de la collision d'un moment orbital électronique non nul, dirigé suivant $\mathbf{N}$, le moment cinétique orbital relatif des deux atomes, et proportionnel à leur vitesse angulaire de rotation $N / I \quad\left(I=\mu r^{2}, \mu\right.$ masse réduite, $r$ distance internucléaire). L'hamiltonien effectif peut s'écrire [9] $\mathscr{H}_{1} \hbar^{-1}=\frac{\gamma(r)}{I} \mathbf{S} . \mathbf{N} ; \quad$ le coefficient $\gamma(r)$ étant sans dimension. En substituant $N \approx \mu \bar{V}_{r} r_{0}$ et $\tau_{\text {coll }} \approx r_{0} / \bar{V}_{r}$ $\left(r_{0}\right.$ distance minimum d'approche pour les chocs de petits paramètres d'impact), on trouve :

$$
\overline{\left|\mathscr{H}_{1}\right|^{2}} \hbar^{-2} \tau_{\text {coll }}^{2} \propto\left|\gamma\left(r_{0}\right)\right|^{2}
$$

c'est-à-dire un résultat qui ne dépend ni de $\mu$ ni de $\bar{V}_{r}$.

Le calcul des sections efficaces fait par Herman [9] ne tient pas compte du spin nucléaire $I$ de l'alcalin et fournit la section efficace $\sigma$ relative à l'observable $\left\langle S_{z}\right\rangle$ pour un isotope de $\mathrm{Rb}$ de $I$ nul. Or, pour une interaction faible de type magnétique, on prévoit [2], [12] que les sections efficaces $\sigma_{n}$ et $\sigma_{e}$ associées aux observables $\left\langle I_{z}\right\rangle$ et $\left\langle Q_{e}\right\rangle$ sont données en fonction de $\sigma$ par les relations :

$$
\begin{gathered}
\sigma_{n}=\frac{\sigma}{(2 I+1)^{2}}\left[j\left(\omega_{F}\right)+j(\Delta W)\right] \\
\sigma_{e}=\sigma\left[\frac{j\left(\omega_{F}\right)-j(\Delta W)}{(2 I+1)^{2}}+j(\Delta W)\right]
\end{gathered}
$$

où $\omega_{F}$ et $\Delta W$ sont les fréquences Zeeman et hyperfine des atomes $\mathrm{Rb}$, et $j(\omega)$ est proportionnel à la densité spectrale de l'interaction aléatoire désorientatrice (définie suivant l'usage comme la transformée de Fourier de la fonction de corrélation).

Si la fonction de corrélation de l'interaction désorientatrice lors des collisions en phase gazeuse possède uniquement comme temps caractéristique la durée d'une collision, $j(\omega)$ est de la forme $1 /\left(1+\omega^{2} \tau_{\text {coll }}^{2}\right)$

TABLEAU III

RAPPORTS ENTRE LES SEGTIONS EFFICAGES DE DÉSORIENTATION : COMPARAISON ENTRE VALEURS THÉORIQUES ET EXPÉRIMENTALES

\begin{tabular}{ccccc} 
& \multicolumn{2}{c}{ VALEURS THÉORIQUES } & \\
\cline { 2 - 3 } & 1 & 2 & & VALEURS \\
& $j\left(\omega_{F}\right)=j(\Delta W)=1$ & $j(\Delta W)=1$ & \\
EXPÉRIMENTALES
\end{tabular}


avec $\tau_{\text {coll }} \approx 10^{-12} \mathrm{~s}$ (temps mis par un atome pour parcourir une distance de quelques $\AA$ à la vitesse thermique). On trouve alors $\omega_{F} \tau_{\text {coll }}, \Delta W \tau_{\text {coll }} \ll 1$ et $j\left(\omega_{F}\right) / j(\Delta W)=1$. En utilisant les relations (14) et (15), on obtient dans cette hypothèse certaines valeurs théoriques pour les rapports entre les sections efficaces $\sigma_{n}$ et $\sigma_{e}$ des isotopes ${ }^{87} \mathrm{Rb}(I=3 / 2)$ et ${ }^{85} \mathrm{Rb}$ $(I=5 / 2)$. Ces valeurs sont portées dans la première colonne du tableau III pour être comparées aux valeurs mesurées (colonne 3 ). Pour les deux premiers rapports, il ne paraît pas y avoir de désaccord significatif. Mais on constate que la valeur mesurée de $\sigma_{e} / \sigma_{n}$ est environ deux fois plus grande que prévu, et ceci pour les deux isotopes du $\mathrm{Rb}$. Le résultat expérimental n'est donc pas en accord avec l'hypothèse d'un temps de corrélation unique de l'ordre de $10^{-12} \mathrm{~s}$.

Par contre, si l'on renonce à faire a priori une hypothèse particulière sur le rapport $j\left(\omega_{F}\right) / j(\Delta W)$, le problème se pose de la manière suivante : existe-t-il des valeurs $j\left(\omega_{F}\right)$ et $j(\Delta W)$ telles qu'il y ait accord entre les valeurs mesurées et les valeurs calculées à l'aide des relations (14) et (15) ? La réponse est oui, si l'on accepte la solution $j\left(\omega_{F}\right) / j(\Delta W) \ll 1$ : les valeurs théoriques correspondant en particulier à $j\left(\omega_{F}\right)=0$, $j(\Delta W)=1$ sont portées dans la colonne $2 \mathrm{du}$ tableau III, on voit que toutes sont compatibles avec les valeurs mesurées. Il serait néanmoins souhaitable de vérifier ce résultat grâce à la mesure des constantes de temps relatives à d'autres observables $(\langle\mathbf{S} . \mathbf{I}\rangle$ par exemple ou bien des grandeurs transversales). On peut montrer que les conditions $j\left(\omega_{F}\right)=0, j(\Delta W)=1$ sont en particulier réalisées si les champs magnétiques aléatoires $\mathbf{H}_{\mathrm{A}}$ et $\mathbf{H}_{\mathrm{B}}$ vus par l'atome alcalin au cours de deux chocs $\mathrm{A}$ et $\mathrm{B}$ successifs, distants en moyenne de l'intervalle de temps $\tau_{v}$, sont anticorrélés (c'est-à-dire tels que $\left\langle\mathbf{H}_{\mathrm{A}} \cdot \mathbf{H}_{\mathrm{B}}\right\rangle=\varepsilon\left\langle\left|\mathbf{H}_{\mathrm{A}}\right|^{2}\right\rangle$ avec $\varepsilon<0$, de l'ordre de l'unité $\left.\left({ }^{1}\right)\right)$. Nous n'apportons pas ici d'interprétation à cette hypothèse : sur le plan théorique, il ne nous apparaît pas en effet qu'une anticorrélation appréciable puisse exister entre deux chocs successifs si l'interaction désorientatrice est l'interaction spinorbite.

On voit qu'il serait très intéressant d'étudier systématiquement $1 / T_{n}^{\prime}$ en fonction du champ magnétique statique dans lequel on opère, de manière à couvrir la zone où $\omega_{F} \approx\left(\tau_{v}\right)^{-1}$. En effet, les résultats expérimentaux actuels nous paraissent insuffisants pour apporter maintenant une conclusion sûre.

(1) Dans la zone explorée expérimentalement jusqu'ici, la suite d'inégalités :

$$
\omega_{F} \ll 1 / \tau_{v} \ll \Delta W \ll 1 / \tau_{\text {coll }}
$$

s'est toujours trouvée réalisée. Il est aussi à noter que la présence d'une interaction magnétique caractérisée par un temps de corrélation long, $\approx \tau_{v}$ (comme il a été trouvé pour la relaxation du $\mathrm{Rb}$ dans le $\mathrm{Kr}$ [14]), ne permettrait pas d'améliorer l'accord entre les résultats théoriques et expérimentaux; au contraire le désaccord serait aggravé.
Enfin, on peut comparer les valeurs absolues des sections efficaces mesurées et calculées pour l'interaction $\mathbf{S} . \mathbf{N}$; on trouve :

$$
\begin{aligned}
& \sigma_{\text {mesuré }}=8,5 \times 10^{-24} \mathrm{~cm}^{2} \\
& \sigma_{\text {calculé }}=3,8 \times 10^{-24} \mathrm{~cm}^{2} .
\end{aligned}
$$

Étant donné les approximations nécessaires pour effectuer le calcul théorique [9] et aussi les incertitudes expérimentales, on peut juger qu'il n'y a pas entre les deux de grave désaccord.

Des mesures de section efficace ont été faites antérieurement par Franz [13] qui a opéré sur du Rb naturel mélangé à ${ }^{4} \mathrm{He}$. On remarque que la section efficace que nous mesurons pour $\left\langle I_{z}\right\rangle$ et l'isotope ${ }^{85} \mathrm{Rb}$ est très voisine de celle qu'a obtenue Franz. Il semble donc que Franz ait observé uniquement la constante de temps longue caractérisant la relaxation de $\left\langle S_{z}\right\rangle$ pour l'isotope le plus abondant (75\% du mélange naturel).

En conclusion, il nous paraît que d'autres expériences sont nécessaires pour élucider complètement le mécanisme de la relaxation du $\mathrm{Rb}$ par collisions sur l'hélium. Les difficultés sont surtout actuellement d'ordre expérimental et tiennent à ce que la section efficace de désorientation $\mathrm{Rb}-\mathrm{He}$ est très faible, ce qui nécessite l'emploi de pressions fortes de gaz. Même à une atmosphère, la relaxation sur $\mathrm{He}$ n'est pas le processus dominant. Nous sommes gênés dans l'analyse des courbes par plusieurs constantes de temps provenant de plusieurs modes de diffusion. En même temps l'élargissement des raies optiques devient supérieur à la structure hyperfine, ce qui rend la détection de $\mathbf{S}$. I difficile. Enfin, la fréquence $1 / \tau_{v}$ des collisions $\mathrm{Rb}-\mathrm{He}$ pour un atome $\mathrm{Rb}$ est si grande à ces pressions qu'il nous est difficile de vérifier si $\tau_{v}$ est un temps de corrélation pour l'interaction désorientatrice en faisant varier le champ statique dans la zone où l'on réalise la condition $\omega_{F} \tau_{v} \approx 1$, car cela nécessite des champs plus forts que ceux que notre montage nous permet d'atteindre. Toutes ces difficultés sont atténuées lorsqu'on envisage la relaxation du $\mathrm{Rb}$ en présence d'un gaz rare plus lourd (les études correspondantes pour $\mathrm{Kr}$ ont été décrites par ailleurs [14]).

Cependant pour l'hélium plusieurs résultats nous paraissent désormais acquis : nous avons mis en évidence dans l'évolution de $\left\langle S_{z}\right\rangle$ deux constantes de temps qui peuvent avoir un sens totalement différent : à pression faible elles sont associées à deux modes de diffusion différents, à pression forte elles sont liées à deux observables différentes. Nos mesures montrent qu'il est important de reconnaître l'existence de ces diverses constantes de temps et leur signification pour éviter des erreurs graves dans la détermination des coefficients de diffusion et des sections efficaces. Enfin, l'identité des sections efficaces pour ${ }^{4} \mathrm{He}$ et ${ }^{3} \mathrm{He}$ est compatible avec l'hypothèse selon laquelle l'interaction désorientatrice prédominante est d'origine 
spin-orbite. L'effet du spin nucléaire est indécelable et donc plus faible qu'il n'était attendu théoriquement. La variation de $\sigma_{n}$ quand on passe d'un isotope de $R b$ à l'autre est comprise dans l'hypothèse d'une interaction désorientatrice de type magnétique faible, de même l'identité de $\sigma_{e}$ pour les deux isotopes. Par contre, pour expliquer le rapport $\sigma_{n} / \sigma_{e}$, on est amené à faire certaines hypothèses sur la fonction de corrélation de l'interaction désorientatrice qu'à ce stade nous ne pouvons justifier théoriquement.

\section{BIBLIOGRAPHIE}

[1] Bouchiat (M. A.) et Grossettête (F.), J. Physique Rad., 1966, 27, 353.

[2] Masnou-Seieuws (F.) et Bouchiat (M. A.), J. Physique Rad., 1967, 28, 406.

[3] Mitcheli, (A. C. G.) et Zemansky (M. W.), Resonance Radiation and Excited Atoms, Cambridge University Press, London, 1961.

[4] Aymar-Fehrenbach (M.), Thèse de $3^{\mathrm{e}}$ cycle, Paris, 1967.

[5] Bouchiat (M. A.) et Brossei (J.), Phys. Rev., 1966, 147, 41, et GrossetTÊTE (F.), J. Physique Rad., 1968, 29, 456.

[6] Cohen-Tannoudji (C.), D.E.S., Paris, 1956.

[7] HIRSCHFELDER (J. O.), CuR'TISS (C. F.) et BIRD (R. B.), Molecular Theory of Gases and Liquids (John Wiley \& Sons Inc., New York, 1954), chapitre 8 , table I M.
[8] BERNSTEIN (R. B.) et MUCKERMAN (J. T.), Theoretical Chemistry Institute, University of Wisconsin, Report no 200, 1967.

[9] Herman (R.), Phys. Rev., 1964, 136, A 1576.

[10] Herman (R.), Phys. Rev., 1965, 137, A 1062.

[11] Gamblin (R. L.) et Carver (T. R.), Phys. Rev., 1965, 138, A 947.

[12] Bouchiat (M. A.), J. Physique Rad., 1963, 24, 379 et 611 .

[13] Franz (F. A.), Phys. Rev., 1965, 139, A 603.

[14] Aymar (M.), Bouchiat (M. A.) et Brossé (J.), Phys. Lett., 1967, 24 A, 753.

Bouchiat (M. A.), Brossei, (J.) et PotTtier (L.), Phys. Rev. Lett., 1967, 19, 817. 